\title{
Antimatter signatures of gravitino dark matter decay
}

\author{
Alejandro Ibarra and David Tran \\ DESY, Theory Group, Notkestrasse 85, D-22603 Hamburg, Germany \\ E-mail: alejandro.ibarra@desy.de and david.tran@desy.de \\ Received 6 May 2008 \\ Accepted 2 June 2008 \\ Published 1 July 2008 \\ Online at stacks.iop.org $/ \mathrm{JCAP} / 2008 / \mathrm{i}=07 / \mathrm{a}=002$ \\ doi:10.1088/1475-7516/2008/07/002
}

\begin{abstract}
The scenario of gravitino dark matter with broken $R$-parity naturally reconciles three paradigms that, albeit very well motivated separately, seem to be in mutual conflict: supersymmetric dark matter, thermal leptogenesis and standard big bang nucleosynthesis. Interestingly, the products of the gravitino decay could be observed, opening the possibility of indirect detection of gravitino dark matter. In this paper, we compute the positron and the antiproton fluxes from gravitino decay. We find that a gravitino with a mass of $m_{3 / 2} \sim 150 \mathrm{GeV}$ and a lifetime of $\tau_{3 / 2} \sim 10^{26} \mathrm{~s}$ could simultaneously explain the EGRET anomaly in the extragalactic diffuse gamma ray background and the HEAT excess in the positron fraction. However, the predicted antiproton flux tends to be too large, although the prediction suffers from large uncertainties and might be compatible with present observations for certain choices of propagation parameters.
\end{abstract}

Keywords: cosmic rays, dark matter, cosmology of theories beyond the SM

ArXiv ePrint: 0804.4596 


\section{Contents}

1. Introduction 2

2. Source term 5

$\begin{array}{ll}\text { 3. Antimatter propagation in the galaxy } & 7\end{array}$

3.1. Positron flux . . . . . . . . . . . . . . . . . . . 9 9

3.2. Antiproton flux . . . . . . . . . . . . . . . . . . 12

4. Conclusions and outlook 15

$\begin{array}{ll}\text { Acknowledgments } & 16\end{array}$

$\begin{array}{ll}\text { References } & 17\end{array}$

\section{Introduction}

Models with local supersymmetry predict the existence of a particle with extremely weak interactions: the gravitino. In contrast to the supersymmetric partners of the standard model particles, whose masses are expected to lie in the electroweak domain, the gravitino can have a mass ranging between a few $\mathrm{eV}$ and several $\mathrm{TeV}$ without conflicting with any laboratory experiment. Therefore, the gravitino can very naturally be the lightest supersymmetric particle (LSP), and if it is sufficiently long-lived, it could constitute the dark matter of the Universe [1].

Gravitinos were produced in the early Universe by scatterings in the thermal plasma, but did not subsequently annihilate due to their extremely weak interactions. Therefore, a relic population of gravitinos is expected in the present Universe with a density given by $[2]$

$$
\Omega_{3 / 2} h^{2} \simeq 0.27\left(\frac{T_{\mathrm{R}}}{10^{10} \mathrm{GeV}}\right)\left(\frac{100 \mathrm{GeV}}{m_{3 / 2}}\right)\left(\frac{m_{\widetilde{\mathrm{g}}}}{1 \mathrm{TeV}}\right)^{2}
$$

where $T_{\mathrm{R}}$ is the reheating temperature of the Universe, $m_{3 / 2}$ is the gravitino mass and $m_{\widetilde{\mathrm{g}}}$ is the gluino mass. In predicting the relic abundance of gravitinos, the main uncertainty arises from our ignorance of the thermal history of the Universe before big bang nucleosynthesis (BBN) and in particular of the reheating temperature after inflation. However, we have strong indications that the Universe was very hot after inflation. Namely, the discovery of neutrino masses about ten years ago provided strong support to leptogenesis as the explanation for the observed baryon asymmetry of the Universe. This mechanism can reproduce the observed baryon asymmetry very naturally if the reheating temperature of the Universe was above $10^{9} \mathrm{GeV}$ [3]. Therefore, following equation (1), the gravitino could constitute the dark matter if $m_{3 / 2} \gtrsim 10 \mathrm{GeV}$ for a gluino mass $m_{\widetilde{\mathrm{g}}} \simeq 1 \mathrm{TeV}$, which is consistent with the assumption that the gravitino is the lightest supersymmetric particle.

Remarkably, the conjectures of a reheating temperature of the Universe larger than $10^{9} \mathrm{GeV}$ and a gravitino mass larger than a few $\mathrm{GeV}$ can naturally solve two of the most 
long-standing problems in cosmology: the nature of the dark matter and the origin of the baryon asymmetry of the Universe. Nevertheless, this picture is not exempt from problems. If $R$-parity is exactly conserved, the next-to-LSP (NSLP) can only decay gravitationally into gravitinos and Standard Model particles with a lifetime

$$
\tau_{\mathrm{NLSP}} \simeq 9 \text { days }\left(\frac{m_{3 / 2}}{10 \mathrm{GeV}}\right)^{2}\left(\frac{150 \mathrm{GeV}}{m_{\mathrm{NLSP}}}\right)^{5} .
$$

Then, the NLSP is typically present during and after big bang nucleosynthesis, jeopardizing the successful predictions of the standard nucleosynthesis scenario. This is in fact the case for the most likely candidates for the NLSP: the lightest neutralino and the right-handed stau (or more generically, any negatively charged particle, such as the chargino). More precisely, when the NLSP is the neutralino, the hadrons produced in the neutralino decays typically dissociate the primordial elements [4], yielding abundances in conflict with observations. On the other hand, when the NLSP is a charged particle, $X^{-}$, the formation of the bound state $\left({ }^{4} \mathrm{He} X^{-}\right)$catalyzes the production of ${ }^{6} \mathrm{Li}[5]$ leading to an overproduction of ${ }^{6} \mathrm{Li}$ by a factor $300-600$ [6].

Although the scenario depicted above is the most widely studied, it is not the only possibility. Indeed, several alternatives have been proposed that yield a thermal history of the Universe consistent with the observed relic density of dark matter, successful leptogenesis and successful big bang nucleosynthesis. For instance, in some specific supersymmetric models the NLSP can be a sneutrino [7] or a stop [8], whose late decays do not substantially affect the predictions of big bang nucleosynthesis. Another possibility is to assume some amount of entropy production after NLSP decoupling, which dilutes the NLSP abundance [9]. Finally, if $R$-parity is not exactly conserved, the NLSP can decay into two Standard Model particles well before the onset of big bang nucleosynthesis, avoiding the BBN constraints altogether [10]. This is the scenario that we will adopt in this paper.

When $R$-parity is not imposed, the superpotential of the Minimal Supersymmetric Standard Model (MSSM) reads [11]

$$
W=W_{R_{\mathrm{p}}}+\frac{1}{2} \lambda_{i j k} L_{i} L_{j} e_{k}^{c}+\lambda_{i j k}^{\prime} L_{i} Q_{j} d_{k}^{c}+\frac{1}{2} \lambda_{i j k}^{\prime \prime} u_{i}^{c} d_{j}^{c} d_{k}^{c}+\mu_{i} L_{i} H_{u},
$$

where $W_{R_{\mathrm{p}}}$ is the familiar superpotential with conserved $R$-parity. Present laboratory experiments very severely restrict the size of the $R$-parity breaking couplings. For instance, when the soft masses are $\sim 100 \mathrm{GeV}$, proton stability requires $\lambda_{11 k}^{\prime} \lambda_{11 k}^{\prime \prime} \lesssim 10^{-27}$, and the non-observation of the lepton flavor violating process $\mu \mathrm{Ti} \rightarrow e \mathrm{Ti}$ requires $\lambda_{1 k 2} \lambda_{k 11}^{\prime} \lesssim 4 \times 10^{-8}$, for $k=1,2,3$. An exhaustive list of the laboratory constraints on the $R$-parity violating couplings can be found in [12].

In addition to the laboratory upper bounds, there also exists an allowed window for the $R$-parity violating Yukawa couplings stemming from cosmology. If the $R$ parity violating interactions had been in thermal equilibrium before the electroweak phase transition, any pre-existing baryon or lepton asymmetry would have been erased. Therefore, successful leptogenesis can only be achieved if the out-of-equilibrium condition $\lambda, \lambda^{\prime}, \lambda^{\prime \prime} \lesssim 10^{-7}$ is satisfied [13]. These bounds are sufficient but not necessary conditions and could be relaxed for some specific flavor structures. On the other hand, successful big bang nucleosynthesis is guaranteed if the NLSP lifetime is shorter than $\sim 10^{3} \mathrm{~s}$, which yields a lower bound on the $R$-parity breaking Yukawa couplings. For instance, when the 
NLSP is a right-handed stau, it can decay via $\widetilde{\tau}_{\mathrm{R}} \rightarrow \mu \nu_{\tau}$ through the coupling $\lambda_{323}$ with lifetime

$$
\tau_{\widetilde{\tau}} \simeq 10^{3} \mathrm{~s}\left(\frac{\lambda_{323}}{10^{-14}}\right)^{-2}\left(\frac{m_{\tilde{\tau}}}{100 \mathrm{GeV}}\right)^{-1} .
$$

Therefore, even a tiny amount of $R$-parity violation, $\lambda_{323} \gtrsim 10^{-14}$, is enough to deplete the population of stau NLSPs at the time of BBN down to harmless levels [10]. A similar argument applies for the case of a neutralino NLSP with analogous conclusions.

When $R$-parity is not exactly conserved, the gravitino LSP is no longer stable. Nevertheless, the gravitino decay rate is doubly suppressed by the Planck mass and by the small $R$-parity violation, yielding $[14,15]$

$$
\tau_{3 / 2} \simeq 10^{23} \mathrm{~s}\left(\frac{\lambda}{10^{-7}}\right)^{-2}\left(\frac{m_{3 / 2}}{100 \mathrm{GeV}}\right)^{-3} .
$$

Therefore, for the range of $R$-parity violating couplings favored by cosmology, $10^{-14} \lesssim$ $\lambda, \lambda^{\prime} \lesssim 10^{-7}$, the gravitino lifetime ranges between $10^{23}$ and $10^{37} \mathrm{~s}$ for $m_{3 / 2}=100 \mathrm{GeV}$, which exceeds the age of the Universe by many orders of magnitude. Hence, even though the gravitino is not absolutely stable, it is stable enough to constitute a viable candidate for being the dark matter of the Universe, while preserving the attractive features of the standard big bang nucleosynthesis scenario and thermal leptogenesis.

Interestingly, the gravitino decay products could be observed as a contribution to the flux of cosmic gamma rays, positrons, antiprotons and neutrinos, opening the possibility of indirect detection of gravitino dark matter. We computed in [16] the gamma ray spectrum from gravitino decay, and we found that the anomaly in the extragalactic gamma ray flux reported by Strong et al between 2 and $10 \mathrm{GeV}$ [17] in the EGRET observations [18] could be qualitatively explained by the decay of gravitino dark matter with a mass of $m_{3 / 2} \simeq 150 \mathrm{GeV}$ and a lifetime of $\tau_{3 / 2} \simeq 1.3 \times 10^{26} \mathrm{~s}^{1}{ }^{1}$ The expected anisotropy in the diffuse gamma ray flux was also found to be consistent with the EGRET observations.

Motivated by this result, in this paper we compute the predicted fluxes of positrons and antiprotons from gravitino decay for the same set of parameters, as independent tests of this scenario ${ }^{2}$. The flux of positrons has been measured by a series of experiments: HEAT [21], CAPRICE [22], MASS [23] and AMS-01 [24]. Clearly, if gravitino decay is the explanation for the extragalactic EGRET anomaly, our predicted positron flux should not exceed the measured one. Although the measurements still suffer from large uncertainties, it is intriguing that they seem to point to an excess of positrons at energies larger than $7 \mathrm{GeV}$, which is precisely the energy range where we expect a contribution of positrons from gravitino decay. On the other hand, the antiproton flux has been measured by BESS [25], IMAX [26] and WiZard/CAPRICE [27]. The measurements do not show any deviation from the predictions from conventional astrophysical models of spallation of cosmic rays on the Milky Way disk. Therefore, the viability of our scenario requires that the total antiproton flux lie below the astrophysical background. Future antimatter

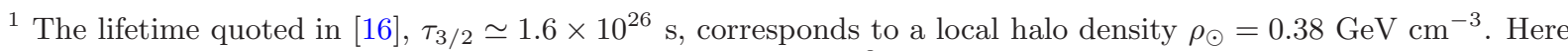
we have adopted the more standard value $\rho_{\odot}=0.30 \mathrm{GeV} \mathrm{cm}^{-3}[19]$. Note that the flux of particles from gravitino decay is proportional to $\rho_{\odot} / \tau_{3 / 2}$; therefore the uncertainty in the value of the local halo density translates into an uncertainty in the gravitino lifetime.

2 The predictions for the neutrino flux will be presented elsewhere [20].
} 
Table 1. Parameters characterizing some commonly used halo models.

\begin{tabular}{lllll}
\hline Halo model & $\alpha$ & $\beta$ & $\gamma$ & $r_{\mathrm{c}}(\mathrm{kpc})$ \\
\hline Navarro, Frenk, White [30] & 1 & 3 & 1 & 20 \\
Isothermal & 2 & 2 & 0 & 3.5 \\
Moore [31] & 1.5 & 3 & 1.5 & 28 \\
\hline
\end{tabular}

experiments such as PAMELA [28] or AMS-02 [29] will provide very precise measurements of the spectra of positrons and antiprotons and will provide important constraints on the scenario of decaying dark matter.

In section 2 we derive the source term for positrons and antiprotons from the decay of gravitinos in the Milky Way halo. Both species, being electrically charged, propagate through the halo in a complicated way that we simulate by means of a conventional diffusion model. In section 3 we discuss and solve the diffusion equation for positrons and antiprotons. Finally, we present our conclusions and an outlook in section 4 .

\section{Source term}

We will assume that the Milky Way dark matter halo is populated by gravitinos with mass $m_{3 / 2}$, their distribution following a density profile $\rho(\vec{r})$, where $\vec{r}$ denotes the position with respect to the center of the Galaxy. The dark matter distribution is usually parametrized as a spherically symmetric profile

$$
\rho(r)=\frac{\rho_{0}}{\left(r / r_{\mathrm{c}}\right)^{\gamma}\left[1+\left(r / r_{\mathrm{c}}\right)^{\alpha}\right]^{(\beta-\gamma) / \alpha}}
$$

where $r=|\vec{r}|$ and the parameters $\alpha, \beta, \gamma$ and $r_{\mathrm{c}}$ are listed in table 1 for some commonly used halo models. Finally, $\rho_{0}$ is a parameter that is adjusted to yield a local halo density of $\rho\left(r_{\odot}\right)=0.30 \mathrm{GeV} \mathrm{cm}^{-3}[19]$, with $r_{\odot}=8.5 \mathrm{kpc}$ being the distance of the Sun to the Galactic center.

Gravitinos at $\vec{r}$ eventually decay with lifetime $\tau_{3 / 2}$ producing antimatter at a rate per unit energy and unit volume given by

$$
Q(E, \vec{r})=\frac{\rho(\vec{r})}{m_{3 / 2} \tau_{3 / 2}} \frac{\mathrm{d} N}{\mathrm{~d} E}
$$

where $\mathrm{d} N / \mathrm{d} E$ is the energy spectrum of antiparticles produced in the decay.

If the gravitino is lighter than the $W^{ \pm}$gauge bosons, the main decay channel is $\psi_{3 / 2} \rightarrow \nu \gamma$, which does not produce antimatter. On the other hand, if it is heavier than the gauge bosons, the decay channels $\psi_{3 / 2} \rightarrow W^{ \pm} \ell^{\mp}$ and $\psi_{3 / 2} \rightarrow Z^{0} \nu$ are kinematically accessible and produce antimatter ${ }^{3}$. Namely, the process $\psi_{3 / 2} \rightarrow W^{-} e^{+}$produces a high energy monoenergetic positron. On the other hand, the antimuon and antitau produced in the processes $\psi_{3 / 2} \rightarrow W^{-} \mu^{+}, W^{-} \tau^{+}$generate a continuous spectrum of positrons in their decays. Lastly, the main contribution comes from the fragmentation of the $W^{ \pm}$and

\footnotetext{
3 The size of the $R$-parity violating couplings for the third generation is expected to be larger than for the first and second generations. Consequently, the charged lepton and the neutrino produced in these decays are expected to have predominantly tau flavor.
} 
Table 2. Branching ratios for gravitino decay in different $R$-parity violating channels for different gravitino masses.

\begin{tabular}{rlll}
\hline$m_{3 / 2}(\mathrm{GeV})$ & $\operatorname{BR}\left(\psi_{3 / 2} \rightarrow \gamma \nu\right)$ & $\operatorname{BR}\left(\psi_{3 / 2} \rightarrow W \ell\right)$ & $\operatorname{BR}\left(\psi_{3 / 2} \rightarrow Z^{0} \nu\right)$ \\
\hline 10 & 1 & 0 & 0 \\
85 & 0.66 & 0.34 & 0 \\
100 & 0.16 & 0.76 & 0.08 \\
150 & 0.05 & 0.71 & 0.24 \\
250 & 0.03 & 0.69 & 0.28 \\
\hline
\end{tabular}

the $Z^{0}$ gauge bosons, which produce a continuous spectrum of positrons (mainly from $\pi^{+}$ decay) and antiprotons that we have obtained using the event generator PYTHIA 6.4 [32]. Thus, the total energy spectrum of antiparticles reads

$$
\frac{\mathrm{d} N}{\mathrm{~d} E} \simeq \operatorname{BR}\left(\psi_{3 / 2} \rightarrow W \ell\right) \frac{\mathrm{d} N^{W \ell}}{\mathrm{d} E}+\mathrm{BR}\left(\psi_{3 / 2} \rightarrow Z^{0} \nu\right) \frac{\mathrm{d} N^{Z \nu}}{\mathrm{d} E}
$$

where $\mathrm{d} N^{W \ell} / \mathrm{d} E$ and $\mathrm{d} N^{Z \nu} / \mathrm{d} E$ denote the energy spectra of antiparticles produced in the indicated decay channel, which depend only on the gravitino mass through the total available energy.

On the other hand, the branching ratios of the relevant decay channels can be straightforwardly computed from the expressions for the gravitino decay rates in [16]. The result is

$$
\begin{aligned}
\operatorname{BR}\left(\psi_{3 / 2} \rightarrow W \ell\right) & =\frac{2\left|U_{\tilde{W} \ell}\right|^{2} f\left(M_{\mathrm{W}} / m_{3 / 2}\right)}{\left|U_{\tilde{\gamma} \nu}\right|^{2}+2\left|U_{\tilde{W} \ell}\right|^{2} f\left(M_{\mathrm{W}} / m_{3 / 2}\right)+\left|U_{\tilde{Z} \nu}\right|^{2} f\left(M_{\mathrm{Z}} / m_{3 / 2}\right)}, \\
\operatorname{BR}\left(\psi_{3 / 2} \rightarrow Z^{0} \nu\right) & =\frac{\left|U_{\tilde{Z} \nu}\right|^{2} f\left(M_{\mathrm{Z}} / m_{3 / 2}\right)}{\left|U_{\tilde{\gamma} \nu}\right|^{2}+2\left|U_{\tilde{W} \ell}\right|^{2} f\left(M_{\mathrm{W}} / m_{3 / 2}\right)+\left|U_{\tilde{Z} \nu}\right|^{2} f\left(M_{\mathrm{Z}} / m_{3 / 2}\right)},
\end{aligned}
$$

where $f(x)=1-(4 / 3) x^{2}+(1 / 3) x^{8}$ and $U_{\tilde{\gamma} \nu}, U_{\tilde{W} \ell}, U_{\tilde{Z} \nu}$ denote the mixings photinoneutrino, charged wino-charged lepton and zino-neutrino, respectively, which satisfy the following relations:

$$
\begin{aligned}
& \left|U_{\widetilde{\gamma} \nu}\right| \simeq\left[\frac{\left(M_{2}-M_{1}\right) \sin \theta_{\mathrm{W}} \cos \theta_{\mathrm{W}}}{M_{1} \cos ^{2} \theta_{\mathrm{W}}+M_{2} \sin ^{2} \theta_{\mathrm{W}}}\right]\left|U_{\widetilde{Z} \nu}\right|, \\
& \left|U_{\widetilde{W} \ell}\right| \simeq \sqrt{2} \cos \theta_{\mathrm{W}} \frac{M_{1} \sin ^{2} \theta_{\mathrm{W}}+M_{2} \cos ^{2} \theta_{\mathrm{W}}}{M_{2}}\left|U_{\widetilde{Z} \nu}\right| .
\end{aligned}
$$

In this expression, $M_{1}$ and $M_{2}$ are the $U(1)_{Y}$ and $S U(2)_{L}$ gaugino masses at low energies and $\theta_{\mathrm{W}}$ is the weak mixing angle. It is commonly assumed that the gaugino masses unify at the Grand Unified Scale, $M_{X}=2 \times 10^{16} \mathrm{GeV}$. Under this assumption, the ratio between the gaugino masses at low energies is predicted to be $M_{2} / M_{1} \simeq 1.9$, which yields

$$
\left|U_{\widetilde{\gamma} \nu}\right|:\left|U_{\widetilde{Z}}\right|:\left|U_{\widetilde{W} \ell}\right| \simeq 1: 3.2: 3.5 .
$$

Therefore, the gravitino branching ratios in the different decay modes depend only on the gravitino mass (see table 2).

We conclude that, under the assumption of gaugino mass universality, the total energy spectrum of antiparticles from gravitino decay, $\mathrm{d} N / \mathrm{d} E$, depends exclusively on 
the gravitino mass. This makes our scenario very predictive: for a given halo model the source term $Q(E, \vec{r})$ depends on only two unknown parameters, namely the gravitino mass and the gravitino lifetime; the former determines the spectral shape of the source function and the latter the normalization.

\section{Antimatter propagation in the galaxy}

Antimatter propagation in the Milky Way is commonly described by a stationary two-zone diffusion model with cylindrical boundary conditions [33]. Under this approximation, the number density of antiparticles per unit kinetic energy, $f(T, \vec{r}, t)$, satisfies the following transport equation, which applies both for positrons and antiprotons:

$0=\frac{\partial f}{\partial t}=\nabla \cdot[K(T, \vec{r}) \nabla f]+\frac{\partial}{\partial T}[b(T, \vec{r}) f]-\nabla \cdot\left[\vec{V}_{\mathrm{c}}(\vec{r}) f\right]-2 h \delta(z) \Gamma_{\mathrm{ann}} f+Q(T, \vec{r})$.

The boundary conditions require the solution $f(T, \vec{r}, t)$ to vanish at the boundary of the diffusion zone, which is approximated by a cylinder with half-height $L=1-15 \mathrm{kpc}$ and radius $R=20 \mathrm{kpc}$.

The first term on the right-hand side of the transport equation is the diffusion term, which accounts for the propagation through the tangled Galactic magnetic field. The diffusion coefficient $K(T, \vec{r})$ is assumed to be constant throughout the diffusion zone and is parametrized by

$$
K(T)=K_{0} \beta \mathcal{R}^{\delta}
$$

where $\beta=v / c$ and $\mathcal{R}$ is the rigidity of the particle, which is defined as the momentum in $\mathrm{GeV}$ per unit charge, $\mathcal{R} \equiv p(\mathrm{GeV}) / Z$. The normalization $K_{0}$ and the spectral index $\delta$ of the diffusion coefficient are related to the properties of the interstellar medium and can be determined from the flux measurements of other cosmic ray species, mainly from the boron to carbon $(\mathrm{B} / \mathrm{C})$ ratio [34]. The second term accounts for energy losses due to inverse Compton scattering on starlight or the cosmic microwave background, synchrotron radiation and ionization. The third term is the convection term, which accounts for the drift of charged particles away from the disk induced by the Milky Way's Galactic wind. It has axial direction and is also assumed to be constant inside the diffusion region: $\vec{V}_{\mathrm{c}}(\vec{r})=V_{\mathrm{c}} \operatorname{sign}(z) \vec{k}$. The fourth term accounts for antimatter annihilation with rate $\Gamma_{\mathrm{ann}}$, when it interacts with ordinary matter in the Galactic disk, which is assumed to be an infinitely thin disk with half-width $h=100$ pc. Lastly, $Q(T, \vec{r})$ is the source term of positrons or antiprotons which was derived in section 2. In this equation, reacceleration effects and non-annihilating interactions of antimatter in the Galactic disk have been neglected.

The solution of the transport equation at the Solar System, $r=r_{\odot}, z=0$, can be formally expressed by the convolution

$$
f(T)=\frac{1}{m_{3 / 2} \tau_{3 / 2}} \int_{0}^{T \max } \mathrm{d} T^{\prime} G\left(T, T^{\prime}\right) \frac{\mathrm{d} N\left(T^{\prime}\right)}{\mathrm{d} T^{\prime}},
$$

where $T_{\max }=m_{3 / 2}$ for the case of the positrons and $T_{\max }=m_{3 / 2}-m_{\mathrm{p}}$ for the antiprotons. The solution is thus factorized into two parts. The first part, given by the Green's function $G\left(T, T^{\prime}\right)$, encodes all of the information about the astrophysics (such as the details of 
the halo profile and the complicated propagation of antiparticles in the Galaxy) and is universal for any decaying dark matter candidate. The remaining part depends exclusively on the nature and properties of the decaying dark matter candidate, namely the mass, the lifetime and the energy spectrum of antiparticles produced in the decay.

Finally, the flux of primary antiparticles at the Solar System from dark matter decay is given by

$$
\Phi^{\operatorname{prim}}(T)=\frac{v}{4 \pi} f(T),
$$

where $v$ is the velocity of the antimatter particle.

In the scenario we are considering the gravitino mass and lifetime are constrained by requiring a qualitatively good agreement of the predicted extragalactic gamma ray flux with the EGRET data: $m_{3 / 2}=150 \mathrm{GeV}$ and $\tau_{3 / 2}=1.3 \times 10^{26} \mathrm{~s}$ [16]. On the other hand, the energy spectrum of antiparticles, $\mathrm{d} N / \mathrm{d} T$, is determined by the well-understood physics of fragmentation. Therefore, the only uncertainties in the computation of the antimatter fluxes stem from the determination of the Green's function, i.e. from the uncertainties in the propagation parameters and the halo profile. As we will see, the uncertainties in the precise shape of the halo profile are not crucial for the determination of the primary antimatter fluxes. On the other hand, the uncertainties in the propagation parameters can substantially change the predictions for the antimatter fluxes, even by two orders of magnitude for the antiproton flux.

The reason for this large uncertainty is a correlation among the diffusion parameters and the size of the diffusion zone. Secondary cosmic rays are produced by spallation of primary cosmic rays in the Galactic disk. Therefore, the measurement of primary and secondary cosmic ray fluxes (particularly the boron to carbon ratio) provides information about the diffusive properties of the interstellar medium. Unfortunately, there exist degeneracies in the determination of the diffusion parameters. For instance, an increase in the size of the diffusion zone, which allows for a longer propagation time of cosmic rays inside the diffusion zone before escaping, can be compensated by a simultaneous increase of the diffusion coefficient, which facilitates a faster diffusion of cosmic rays away from the Galactic disk. However, this degeneracy does not hold for the antimatter fluxes from decaying dark matter, since antimatter is not only produced in the Galactic disk, but also in the whole dark matter halo. Therefore, an increase in the size of the diffusion zone translates into an increase in the number of injected primary antiparticles, which is not compensated by the simultaneous increase of the diffusion coefficient. As a result, the antimatter fluxes from decaying dark matter can vary substantially for the range of astrophysical parameters which successfully reproduce the secondary cosmic ray fluxes. The ranges of the astrophysical parameters that are consistent with the $\mathrm{B} / \mathrm{C}$ ratio and that produce the maximal, median and minimal positron and antiproton fluxes are listed in tables 3 and $5[35]^{4}$.

Positrons and antiprotons have different properties and their respective transport equations can be approximated by different limits of equation (12), thus allowing simple analytic solutions. Let us discuss each case separately.

${ }^{4}$ It is interesting to note that measurements of the ratio of stable to unstable secondary cosmic rays, such as ${ }^{10} \mathrm{Be} /{ }^{9} \mathrm{Be},{ }^{36} \mathrm{Cl} / \mathrm{Cl}$ and ${ }^{26} \mathrm{Al} /{ }^{27} \mathrm{Al}$, break the above-mentioned degeneracy [36]. However, present experiments only lead to a slight improvement in the determination of the propagation parameters which do not affect substantially the predictions for the antimatter fluxes. 
Table 3. Astrophysical parameters compatible with the $\mathrm{B} / \mathrm{C}$ ratio that yield the minimum (M2), median (MED) and maximal (M1) flux of positrons.

\begin{tabular}{lllc}
\hline Model & $\delta$ & $K_{0}\left(\mathrm{kpc}^{2} \mathrm{Myr}^{-1}\right)$ & $L(\mathrm{kpc})$ \\
\hline M2 & 0.55 & 0.00595 & 1 \\
MED & 0.70 & 0.0112 & 4 \\
M1 & 0.46 & 0.0765 & 15 \\
\hline
\end{tabular}

\subsection{Positron flux}

For the case of the positrons, Galactic convection and annihilations in the disk can be neglected in the transport equation, which is then simplified to

$$
\nabla \cdot\left[K(T, \vec{r}) \nabla f_{e^{+}}\right]+\frac{\partial}{\partial T}\left[b(T, \vec{r}) f_{e^{+}}\right]+Q(T, \vec{r})=0,
$$

where the rate of energy loss, $b(T, \vec{r})$, is assumed to be a spatially constant function parametrized by $b(T)=T^{2} /\left(T_{0} \tau_{\mathrm{E}}\right)$, with $T_{0}=1 \mathrm{GeV}$ and $\tau_{\mathrm{E}}=10^{16} \mathrm{~s}$.

The solution to this equation is formally given by the convolution equation (14). The explicit form of the Green's function is [37]

$$
G_{e^{+}}\left(T, T^{\prime}\right)=\sum_{n, m=1}^{\infty} B_{n m}\left(T, T^{\prime}\right) J_{0}\left(\zeta_{n} \frac{r_{\odot}}{R}\right) \sin \left(\frac{m \pi}{2}\right),
$$

where $J_{0}$ is the zeroth-order Bessel function of the first kind, whose successive zeros are denoted by $\zeta_{n}$. On the other hand,

$B_{n m}\left(T, T^{\prime}\right)=\frac{\tau_{\mathrm{E}} T_{0}}{T^{2}} C_{n m} \exp \left\{\left(\frac{\zeta_{n}^{2}}{R^{2}}+\frac{m^{2} \pi^{2}}{4 L^{2}}\right) \frac{K_{0} \tau_{\mathrm{E}}}{\delta-1}\left[\left(\frac{T}{T_{0}}\right)^{\delta-1}-\left(\frac{T^{\prime}}{T_{0}}\right)^{\delta-1}\right]\right\}$,

with

$$
C_{n m}=\frac{2}{J_{1}^{2}\left(\zeta_{n}\right) R^{2} L} \int_{0}^{R} r^{\prime} \mathrm{d} r^{\prime} \int_{-L}^{L} \mathrm{~d} z^{\prime} \rho\left(\vec{r}^{\prime}\right) J_{0}\left(\zeta_{n} \frac{r^{\prime}}{R}\right) \sin \left[\frac{m \pi}{2 L}\left(L-z^{\prime}\right)\right],
$$

where $J_{1}$ is the first-order Bessel function.

The Green's function can be well approximated by the following interpolating function, which is valid for any decaying dark matter particle:

$$
G_{e^{+}}\left(T, T^{\prime}\right) \simeq \frac{10^{16}}{T^{2}} \mathrm{e}^{a+b\left(T^{\delta-1}-T^{\prime \delta-1}\right)} \theta\left(T^{\prime}-T\right) \mathrm{cm}^{-3} \mathrm{~s},
$$

where $T$ and $T^{\prime}$ are expressed in units of GeV. The coefficients $a$ and $b$ can be found in table 4 for the NFW profile and the different diffusion models listed in table 3 . This approximation works better than a 15-20\% over the whole range of energies. We find numerically that the Green's function is not very sensitive to the choice of the halo profile; therefore the corresponding coefficients can be well approximated by table 4 .

The interstellar positron flux from gravitino decay can be computed from equations (14) and (15), the result being

$$
\Phi_{e^{+}}^{\mathrm{prim}}(T)=\frac{c}{4 \pi m_{3 / 2} \tau_{3 / 2}} \int_{0}^{m_{3 / 2}} \mathrm{~d} T^{\prime} G_{e^{+}}\left(T, T^{\prime}\right) \frac{\mathrm{d} N_{e^{+}}\left(T^{\prime}\right)}{\mathrm{d} T^{\prime}} .
$$



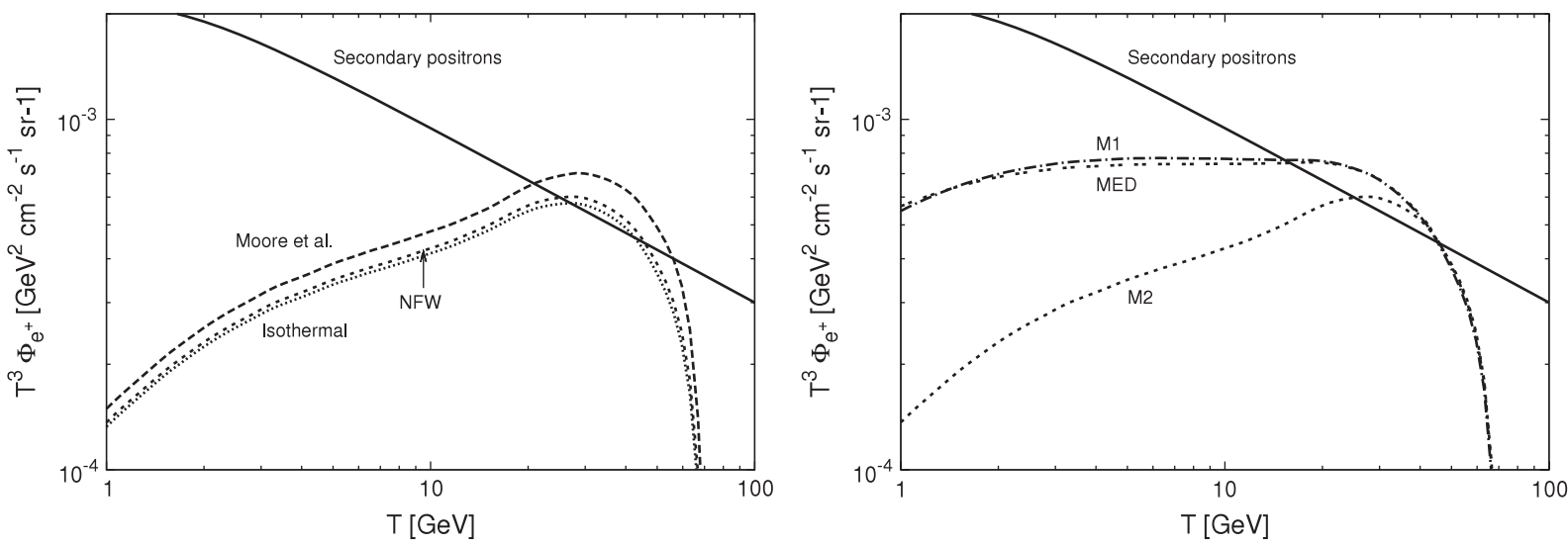

Figure 1. Interstellar positron flux from the decay of gravitinos with $m_{3 / 2} \simeq$ $150 \mathrm{GeV}$ and $\tau_{3 / 2} \simeq 1.3 \times 10^{26} \mathrm{~s}$. In the left plot we assume the M2 diffusion model (see table 3) and we study the sensitivity of the positron flux to various halo profiles. On the other hand, in the right plot we assume a NFW halo profile and we study the sensitivity of the positron flux to the diffusion model. We also show for comparison the secondary positron flux from spallation of cosmic rays on the Galactic disk.

Table 4. Coefficients of the interpolating function equation (20) for the positron Green's function, assuming a NFW halo profile and for the different diffusion models in table 3 .

\begin{tabular}{llc}
\hline Model & $a$ & $b$ \\
\hline M2 & -0.9716 & -10.012 \\
MED & -1.0203 & -1.4493 \\
M1 & -0.9809 & -1.1456 \\
\hline
\end{tabular}

Table 5. Astrophysical parameters compatible with the $\mathrm{B} / \mathrm{C}$ ratio that yield the minimal (MIN), median (MED) and maximal (MAX) flux of antiprotons.

\begin{tabular}{lllcl}
\hline Model & $\delta$ & $K_{0}\left(\mathrm{kpc}^{2} \mathrm{Myr}^{-1}\right)$ & $L(\mathrm{kpc})$ & $V_{\mathrm{c}}\left(\mathrm{km} \mathrm{s}^{-1}\right)$ \\
\hline MIN & 0.85 & 0.0016 & 1 & 13.5 \\
MED & 0.70 & 0.0112 & 4 & 12 \\
MAX & 0.46 & 0.0765 & 15 & 5 \\
\hline
\end{tabular}

We show in figure 1 the predicted interstellar positron flux from gravitino decay for different halo profiles (left plot) and for different diffusion models (right plot). As expected, the dependence of the positron flux on the choice of the halo model is quite weak. On the other hand, the dependence on the diffusion model is important only at low energies, where the signal lies well below the background. At energies where the contribution to the total positron flux from gravitino decay can be visible, $T \gtrsim 7 \mathrm{GeV}$, the choice of the diffusion model only changes the primary positron flux by a factor 2-3.

Rather than measuring the positron flux, most experiments measure the positron fraction, $\Phi_{e^{+}} /\left(\Phi_{e^{-}}+\Phi_{e^{+}}\right)$, since most sources of systematic error, such as detector 

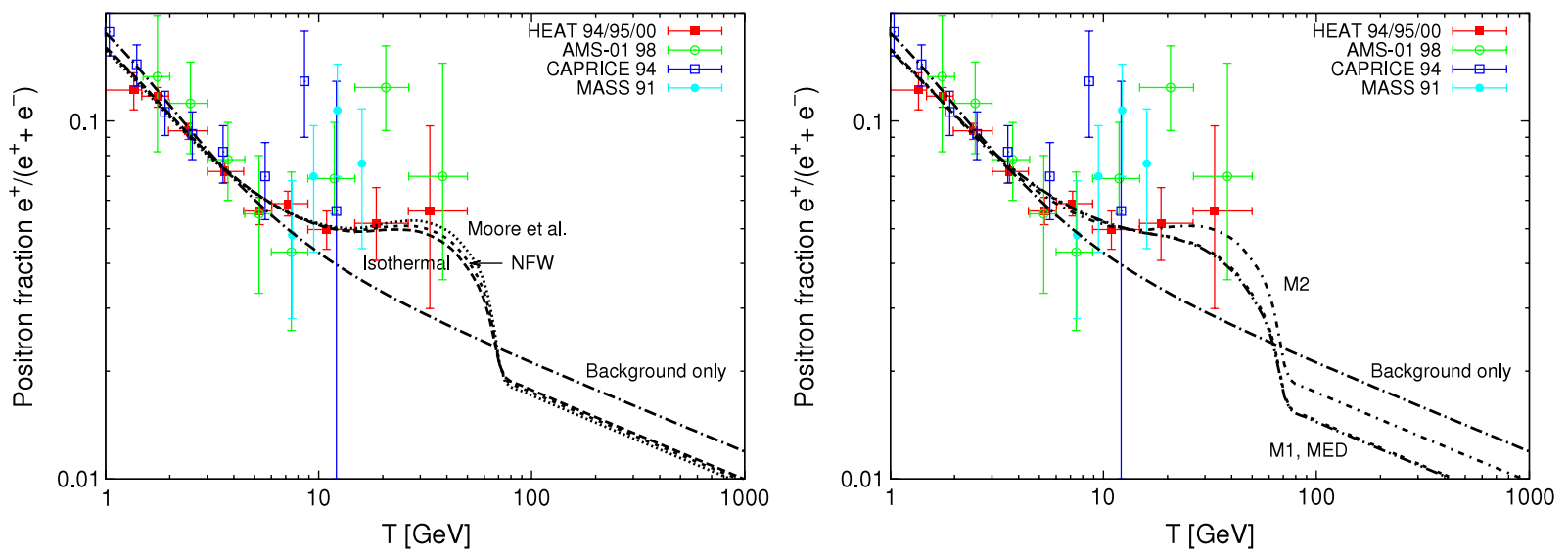

Figure 2. Same as figure 1, but for the positron fraction.

acceptance or trigger efficiency, cancel out when computing the ratio of particle fluxes. Furthermore, the effects of solar modulation, which are important in computing the positron flux at the top of the atmosphere below $10 \mathrm{GeV}$, also cancel out in the positron fraction when solar modulation is assumed to be charge-sign independent. In addition to the primary positron flux from gravitino decay there exists a secondary positron flux originating from the collision of primary protons and other nuclei on the interstellar medium, which constitutes the background to our signal. For the background fluxes of primary and secondary electrons, as well as secondary positrons, we use the parametrizations obtained in [38] from detailed computer simulations of cosmic ray propagation [39]:

$$
\begin{aligned}
& \Phi_{e^{-}}^{\text {prim }}(T)=\frac{0.16 T^{-1.1}}{1+11 T^{0.9}+3.2 T^{2.15}}\left(\mathrm{GeV}^{-1} \mathrm{~cm}^{-2} \mathrm{~s}^{-1} \mathrm{sr}^{-1}\right), \\
& \Phi_{e^{-}}^{\sec }(T)=\frac{0.70 T^{0.7}}{1+110 T^{1.5}+600 T^{2.9}+580 T^{4.2}}\left(\mathrm{GeV}^{-1} \mathrm{~cm}^{-2} \mathrm{~s}^{-1} \mathrm{sr}^{-1}\right), \\
& \Phi_{e^{+}}^{\sec }(T)=\frac{4.5 T^{0.7}}{1+650 T^{2.3}+1500 T^{4.2}}\left(\mathrm{GeV}^{-1} \mathrm{~cm}^{-2} \mathrm{~s}^{-1} \mathrm{sr}^{-1}\right),
\end{aligned}
$$

where $T$ is expressed in units of GeV. Then, the positron fraction reads

$$
\operatorname{PF}(T)=\frac{\Phi_{e^{+}}^{\mathrm{prim}}(T)+\Phi_{e^{+}}^{\mathrm{sec}}(T)}{\Phi_{e^{+}}^{\mathrm{prim}}(T)+\Phi_{e^{+}}^{\mathrm{sec}}(T)+k \Phi_{e^{-}}^{\mathrm{prim}}(T)+\Phi_{e^{-}}^{\mathrm{sec}}(T)},
$$

where following $[38,40]$ we have left the normalization of the primary electron flux as a free parameter, $k$, to be fitted in order to match the observations of the positron fraction. When there is no primary source of positrons, the positron fraction is best fitted for $k=0.88[40]$.

We show in figure 2 the positron fraction for different halo profiles (left plot) and for different diffusion models (right plot). In accordance with the results for the primary positron flux, the dependence of the positron fraction on the halo model is very weak. Furthermore, the mild dependence of the primary positron flux on the choice of the diffusion model becomes even milder when computing the positron fraction. The reason 
for this is double: firstly, the primary positron flux is never much larger than the secondary positron flux, and secondly, the dependence on the choice of diffusion model is partially absorbed by the normalization of the primary electron flux that we have left as a free parameter. Note also that the M2 model, which produces the minimal primary positron flux, yields the most prominent bump in the positron fraction. This 'inversion' is again a consequence of having left the normalization of the primary electron flux as a free parameter. In order to reproduce the measured positron fraction at low energies, the normalization of the primary electron flux $k$ has to be smaller in the M2 model than in the MED and M1 (the precise values are $k=1.07,1.28,1.29$ for the M2, MED and M1 model respectively). Then, with the primary positron flux being comparable for all the diffusion models at energies above $\sim 10 \mathrm{GeV}$, the smaller value of $k$ for the M2 model yields a larger positron flux in this energy range than for the M1 and MED models.

In conclusion, we find that gravitino parameters which predict a departure from a simple power law in the extragalactic gamma ray spectrum at energies above $2 \mathrm{GeV}$ (as observed by EGRET) inevitably predict a bump in the positron fraction at energies above $7 \mathrm{GeV}$ (as observed by HEAT). Furthermore, the presence of this feature is not very sensitive to the many astrophysical uncertainties. This remarkable result holds not only for the scenario of gravitino dark matter with broken $R$-parity, but also for any other scenario of decaying dark matter with lifetime $\sim 10^{26} \mathrm{~s}$ which decays predominantly into $Z^{0}$ and/or $W^{ \pm}$gauge bosons with momentum $\sim 50 \mathrm{GeV}$.

\subsection{Antiproton flux}

The general transport equation, equation (12), can be simplified by taking into account that energy losses are negligible for antiprotons. Therefore, the transport equation for the antiproton density, $f_{\bar{p}}(T, \vec{r}, t)$, is simplified to

$0=\frac{\partial f_{\bar{p}}}{\partial t}=\nabla \cdot\left(K(T, \vec{r}) \nabla f_{\bar{p}}\right)-\nabla \cdot\left(\vec{V}_{\mathrm{c}}(\vec{r}) f_{\bar{p}}\right)-2 h \delta(z) \Gamma_{\mathrm{ann}} f_{\bar{p}}+Q(T, \vec{r})$,

where the annihilation rate, $\Gamma_{\text {ann }}$, is given by

$$
\Gamma_{\text {ann }}=\left(n_{\mathrm{H}}+4^{2 / 3} n_{\mathrm{He}}\right) \sigma_{\bar{p} p}^{\mathrm{ann}} v_{\bar{p}} .
$$

In this expression it has been assumed that the annihilation cross section of an antiproton and a helium nucleus is related to the annihilation cross section of an antiproton and a proton by the simple geometrical factor $4^{2 / 3}$. On the other hand, $n_{\mathrm{H}} \sim 1 \mathrm{~cm}^{-3}$ is the number density of hydrogen nuclei in the Milky Way disk, $n_{\mathrm{He}} \sim 0.07 n_{\mathrm{H}}$ the number density of helium nuclei and $\sigma_{\bar{p} p}^{\text {ann }}$ is the annihilation cross section, which is parametrized by $[41]$

$\sigma_{\bar{p} p}^{\mathrm{ann}}(T)= \begin{cases}661\left(1+0.0115 T^{-0.774}-0.948 T^{0.0151}\right) \mathrm{mb}, & T<15.5 \mathrm{GeV}, \\ 36 T^{-0.5} \mathrm{mb}, & T \geq 15.5 \mathrm{GeV} .\end{cases}$

Analogously to the positron case, the solution to the transport equation can be expressed as a convolution of the form equation (14). The analytic expression for the Green's function reads [42]

$G_{\bar{p}}\left(T, T^{\prime}\right)=\sum_{i=1}^{\infty} \exp \left(-\frac{V_{\mathrm{c}} L}{2 K(T)}\right) \frac{y_{i}(T)}{A_{i}(T) \sinh \left(S_{i}(T) L / 2\right)} J_{0}\left(\zeta_{i} \frac{r_{\odot}}{R}\right) \delta\left(T-T^{\prime}\right)$, 
Table 6. Coefficients of the interpolating function equation (33) for the antiproton Green's function for the NFW halo profile.

\begin{tabular}{lrrr}
\hline Model & \multicolumn{1}{c}{$x$} & \multicolumn{1}{l}{$y$} & \multicolumn{1}{c}{$z$} \\
\hline MIN & -0.0537 & 0.7052 & -0.1840 \\
MED & 1.8002 & 0.4099 & -0.1343 \\
MAX & 3.3602 & -0.1438 & -0.0403 \\
\hline
\end{tabular}

where

$$
\begin{gathered}
y_{i}(T)=\frac{4}{J_{1}^{2}\left(\zeta_{i}\right) R^{2}} \int_{0}^{R} r^{\prime} \mathrm{d} r^{\prime} J_{0}\left(\zeta_{i} \frac{r^{\prime}}{R}\right) \int_{0}^{L} \mathrm{~d} z^{\prime} \exp \left(\frac{V_{\mathrm{c}}\left(L-z^{\prime}\right)}{2 K(T)}\right) \\
\times \sinh \left(\frac{S_{i}\left(L-z^{\prime}\right)}{2}\right) \rho\left(\vec{r}^{\prime}\right),
\end{gathered}
$$

and

$$
\begin{aligned}
& A_{i}(T)=2 h \Gamma_{\mathrm{ann}}(T)+V_{\mathrm{c}}+k S_{i}(T) \operatorname{coth} \frac{S_{i}(T) L}{2}, \\
& S_{i}(T)=\sqrt{\frac{V_{\mathrm{c}}^{2}}{K(T)^{2}}+\frac{4 \zeta_{i}^{2}}{R^{2}}} .
\end{aligned}
$$

We find that the Green's function can be numerically approximated by the following interpolation function:

$$
G_{\bar{p}}\left(T, T^{\prime}\right) \simeq 10^{14} \mathrm{e}^{x+y \ln T+z \ln ^{2} T} \delta\left(T^{\prime}-T\right) \mathrm{cm}^{-3} \mathrm{~s},
$$

which, again, is valid for any decaying dark matter particle. The coefficients $x, y$ and $z$ for the NFW profile can be found in table 6 for the various diffusion models in table 5 . In this case the approximation is accurate to $5-10 \%$. As in the case of the positrons, the dependence of the Green's function on the halo model is fairly weak.

The interstellar antiproton flux is then given by

$$
\Phi_{\bar{p}}^{\mathrm{IS}}(T)=\frac{v_{\bar{p}}(T)}{4 \pi m_{3 / 2} \tau_{3 / 2}} \int_{0}^{m_{3 / 2}-m_{\mathrm{p}}} \mathrm{d} T^{\prime} G_{\bar{p}}\left(T, T^{\prime}\right) \frac{\mathrm{d} N_{\bar{p}}\left(T^{\prime}\right)}{\mathrm{d} T^{\prime}} .
$$

However, this is not the antiproton flux measured by balloon or satellite experiments, which is affected by solar modulation. In the force field approximation [43] the effect of solar modulation can be included by applying the following simple formula that relates the antiproton flux at the top of the Earth's atmosphere and the interstellar antiproton flux [44]:

$$
\Phi_{\bar{p}}^{\mathrm{TOA}}\left(T_{\mathrm{TOA}}\right)=\left(\frac{2 m_{\mathrm{p}} T_{\mathrm{TOA}}+T_{\mathrm{TOA}}^{2}}{2 m_{\mathrm{p}} T_{\mathrm{IS}}+T_{\mathrm{IS}}^{2}}\right) \Phi_{\bar{p}}^{\mathrm{IS}}\left(T_{\mathrm{IS}}\right)
$$

where $T_{\mathrm{IS}}=T_{\mathrm{TOA}}+\phi_{F}$, with $T_{\mathrm{IS}}$ and $T_{\mathrm{TOA}}$ being the antiproton kinetic energies at the heliospheric boundary and at the top of the Earth's atmosphere, respectively, and $\phi_{F}$ being the solar modulation parameter, which varies between $500 \mathrm{MV}$ and 1.3 GV over the eleven-year solar cycle. Since experiments are usually undertaken near solar minimum 

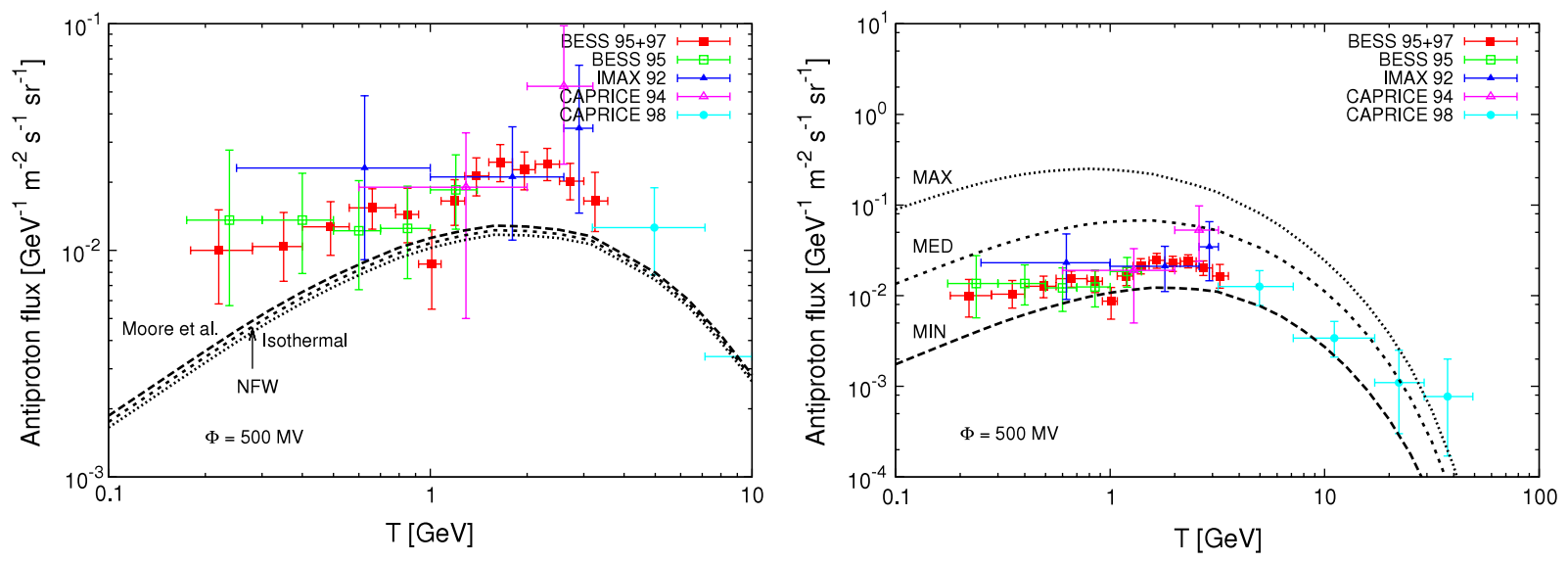

Figure 3. Same as figure 1, but for the primary antiproton flux at the top of the atmosphere. In the left plot the MIN diffusion model was assumed (see table 5).

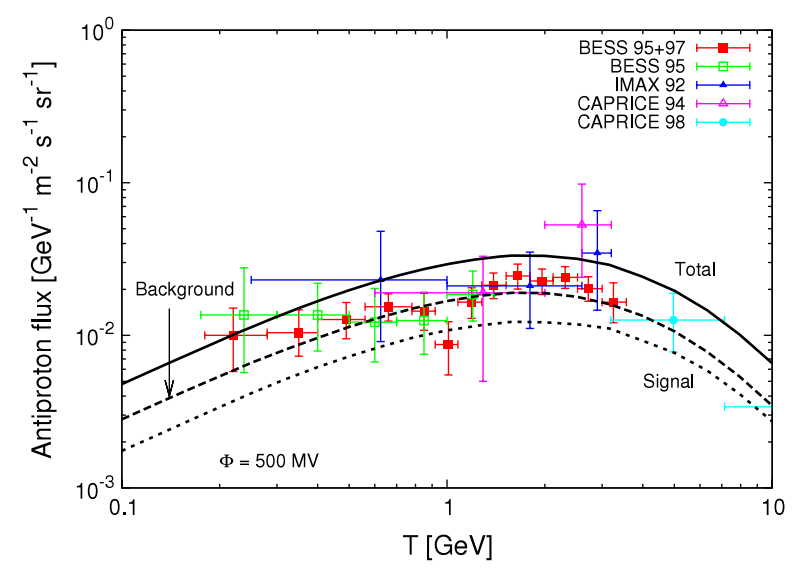

Figure 4. Contributions to the total antiproton flux in the MIN diffusion model.

activity, we will choose $\phi_{F}=500 \mathrm{MV}$ for our numerical analysis in order to compare our predicted flux with the collected data.

We show in figure 3, left plot, the predicted antiproton flux for different halo models. As in the case of the positrons, the sensitivity of the primary antiproton flux to the choice of halo model is fairly mild. We also show in the right plot the predicted antiproton flux from gravitino decay for the diffusion models listed in table 5. From the plot, the extreme sensitivity of the primary antiproton flux to the choice of the diffusion model is apparent: parameters that successfully reproduce the observed B/C ratio lead to antiproton fluxes that span over two orders of magnitude. For a wide range of propagation parameters, the total antiproton flux is well above the observations and thus our scenario is most likely excluded, in spite of all the simplifying assumptions in the diffusion model. However, the MIN model yields a primary flux that is below the measured flux and thus might be compatible with observations.

We have analyzed more carefully the predictions for the MIN model by computing the total antiproton flux. The result is shown in figure 4, where, for consistency, we have 
adopted as background the secondary antiproton flux calculated in [42] for the same MIN model. Although the primary antiproton flux is smaller than the measured one, the total antiproton flux is a factor of two above the observations. Nevertheless, in view of all the uncertainties that enter in the calculation of the antiproton flux, it might be premature to conclusively rule out the scenario of decaying gravitino dark matter. Namely, in addition to the uncertainties stemming from degeneracies in the diffusion parameters, there are also uncertainties from the nuclear cross sections and, to a lesser extent, uncertainties from the description of the interstellar medium and solar modulation (for a discussion of the various uncertainties see [42]). Furthermore, we used a simplified diffusion model that neglects the effects of reacceleration, energy losses and tertiary contributions. Therefore, there could be certain choices of parameters or more refined diffusion models where the total antiproton flux is consistent with experiments ${ }^{5}$.

\section{Conclusions and outlook}

In this paper we have calculated the positron and antiproton fluxes from gravitino dark matter decay. The source term merely depends on two parameters, the gravitino mass and the gravitino lifetime, rendering a very predictive scenario from the particle physics point of view. The main uncertainties arise from the astrophysics, namely from our ignorance of the precise shape of the halo profile and especially from the degeneracies in the determination of the diffusion parameters.

By requiring a qualitatively good agreement of the predicted extragalactic gamma ray flux to the EGRET data, we have fixed $m_{3 / 2}=150 \mathrm{GeV}$ and $\tau_{3 / 2}=1.3 \times 10^{26} \mathrm{~s}$. This choice of parameters completely fixes the source term, and the only indeterminacy in the computation of the antimatter fluxes stems from the unknown astrophysical parameters. Remarkably, with independence of the astrophysical uncertainties, we predict a bump in the positron fraction at energies above $7 \mathrm{GeV}$, in agreement with the HEAT observations. On the other hand, the predicted antiproton flux tends to be too large, although for certain choices of the propagation parameters the predicted flux might also be in agreement with observations.

The main conclusion of this paper is summarized in the three plots in figure 5. There, we show the predicted extragalactic gamma ray flux, positron fraction and antiproton flux compared to the EGRET, HEAT and BESS data respectively, for $m_{3 / 2}=150 \mathrm{GeV}, \tau_{3 / 2}=$ $1.3 \times 10^{26} \mathrm{~s}$ and the MIN diffusion model in table 5 . It is intriguing that for this diffusion model the scenario of gravitino dark matter with broken $R$-parity can qualitatively explain the anomalies observed in the extragalactic gamma ray flux and the positron fraction in a very natural way. It should also be stressed that this scenario was not devised to explain the anomalies in the cosmic ray fluxes, but to reconcile the clashing paradigms of supersymmetric dark matter, thermal leptogenesis and big bang nucleosynthesis. At the same time, we find that the total antiproton flux is slightly larger than the observed one. However, given all the uncertainties that enter in the calculation of the antiproton flux, it might be premature to rule out the present scenario on the basis of this small excess.

To conclude on the phenomenological viability of this scenario, it would be worthwhile to elaborate on the propagation of positrons and especially antiprotons from gravitino

\footnotetext{
${ }^{5}$ Some works have reported a deficit in the predicted secondary antiprotons compared to the observations and argued that this deficit could be connected with a contribution of primary antiprotons [45].
} 

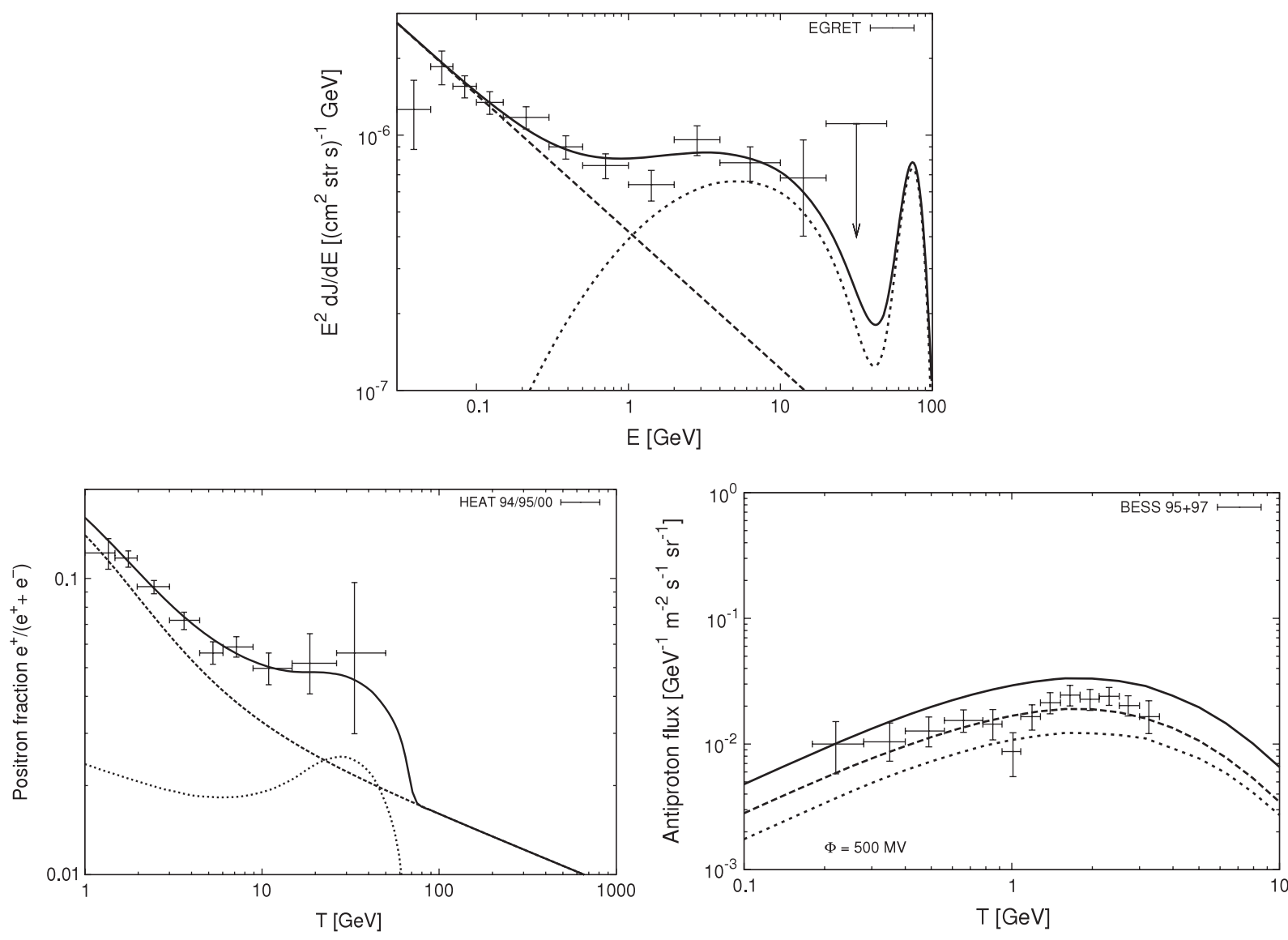

Figure 5. Summary of the signatures of gravitino dark matter decay in the extragalactic gamma ray flux (top), the positron fraction (bottom left) and the antiproton flux (bottom right), compared to the EGRET, HEAT and BESS data respectively. In these plots, we have adopted the MIN diffusion model (see table 5), $m_{3 / 2} \simeq 150 \mathrm{GeV}$ and $\tau_{3 / 2} \simeq 1.3 \times 10^{26} \mathrm{~s}$.

decay by going beyond the simplified diffusion model used in this paper [46]. On the experimental side, the upcoming gamma ray experiment GLAST and the antimatter experiment PAMELA will provide in the near future measurements of the cosmic ray fluxes with unprecedented accuracy, thus providing invaluable information about the scenario of decaying gravitino dark matter.

Finally, we would like to mention that these results are not peculiar to the scenario of gravitino dark matter with broken $R$-parity. The characteristic feature of our scenario is that the dark matter decays at late times into gauge bosons, eventually producing photons, positrons and antiprotons in the fragmentation. Therefore, similar signatures can be expected for other decaying dark matter particles that couple to the $Z^{0}$ and/or $W^{ \pm}$gauge bosons.

\section{Acknowledgments}

We are grateful to Wilfried Buchmüller, Marco Cirelli, Laura Covi, Michael Grefe and Piero Ullio for interesting conversations. 


\section{References}

[1] Pagels H and Primack J R, 1982 Phys. Rev. Lett. 48223 [SPIRES]

[2] Bolz M, Brandenburg A and Buchmüller W, 2001 Nucl. Phys. B 606518 [SPIRES] Bolz M, Brandenburg A and Buchmüller W, 2008 Nucl. Phys. B 790336 (erratum) Pradler J and Steffen F D, 2007 Phys. Rev. D 75023509 [SPIRES] Rychkov V S and Strumia A, 2007 Phys. Rev. D 75075011 [SPIRES]

[3] Davidson S and Ibarra A, 2002 Phys. Lett. B 53525 [SPIRES] Buchmüller W, Di Bari P and Plümacher M, 2005 Ann. Phys., NY 315305 [SPIRES] Giudice G F, Notari A, Raidal M, Riotto A and Strumia A, 2004 Nucl. Phys. B 68589 [SPIRES]

[4] Kawasaki M, Kohri K and Moroi T, 2005 Phys. Rev. D 71083502 [SPIRES]

[5] Pospelov M, 2007 Phys. Rev. Lett. 98231301 [SPIRES]

[6] Hamaguchi K, Hatsuda T, Kamimura M, Kino Y and Yanagida T T, 2007 Phys. Lett. B 650268 [SPIRES]

[7] Kanzaki T, Kawasaki M, Kohri K and Moroi T, 2007 Phys. Rev. D 75025011 [SPIRES]

[8] Diaz-Cruz J L, Ellis J R, Olive K A and Santoso Y, 2007 J. High Energy Phys. JHEP05(2007)003 [SPIRES]

[9] Pradler J and Steffen F D, 2007 Phys. Lett. B 648224 [SPIRES]

[10] Buchmüller W, Covi L, Hamaguchi K, Ibarra A and Yanagida T, 2007 J. High Energy Phys. JHEP03(2007)037 [SPIRES]

[11] For a review, see: Barbier R et al, 2005 Phys. Rep. 4201 [SPIRES]

[12] Allanach B C, Dedes A and Dreiner H K, 1999 Phys. Rev. D 60075014 [SPIRES]

[13] Campbell B A, Davidson S, Ellis J R and Olive K A, 1992 Phys. Lett. B 297118 [SPIRES] Fischler W, Giudice G F, Leigh R G and Paban S, 1991 Phys. Lett. B 25845 [SPIRES] Dreiner H K and Ross G G, 1993 Nucl. Phys. B 410188 [SPIRES]

[14] Takayama F and Yamaguchi M, 2000 Phys. Lett. B 485388 [SPIRES]

[15] See also: Bertone G, Buchmuller W, Covi L and Ibarra A, 2007 J. Cosmol. Astropart. Phys. JCAP11(2007)003 [SPIRES]

Lola S, Osland P and Raklev A R, 2007 Phys. Lett. B 65683 [SPIRES]

[16] Ibarra A and Tran D, 2008 Phys. Rev. Lett. 100061301 [SPIRES]

[17] Strong A W, Moskalenko I V and Reimer O, 2004 Astrophys. J. 613962 [SPIRES] Strong A W, Moskalenko I V and Reimer O, 2004 Astrophys. J. 613956 [SPIRES]

[18] Sreekumar P et al (EGRET Collaboration), 1998 Astrophys. J. 494523 [SPIRES]

[19] Bergstrom L, Ullio P and Buckley J H, 1998 Astropart. Phys. 9137 [SPIRES]

[20] Covi L, Grefe M, Ibarra A and Tran D, 2008 in preparation

[21] Barwick S W et al (HEAT Collaboration), 1997 Astrophys. J. 482 L191 [SPIRES]

[22] Boezio M et al (CAPRICE Collaboration), 2000 Astrophys. J. 532653 [SPIRES]

[23] Grimani C et al, 2002 Astron. Astrophys. 392287 [SPIRES]

[24] Aguilar M et al (AMS-01 Collaboration), 2007 Phys. Lett. B 646145 [SPIRES]

[25] Orito S et al (BESS Collaboration), 2000 Phys. Rev. Lett. 841078 [SPIRES] Matsunaga $\mathrm{H}$ et al, 1998 Phys. Rev. Lett. 814052 [SPIRES]

[26] Mitchell J W et al, 1996 Phys. Rev. Lett. 763057 [SPIRES]

[27] Boezio M et al (WIZARD Collaboration), 1997 Astrophys. J. 487415 [SPIRES] Boezio M et al (WiZard/CAPRICE Collaboration), 2001 Astrophys. J. 561787 [SPIRES]

[28] Picozza P et al, 2007 Astropart. Phys. 27296 [SPIRES] See also the Web page http://pamela.roma2.infn.it/index.php

[29] Barao F (AMS-02 Collaboration), 2004 Nucl. Instrum. Meth. A 535134 [SPIRES] See also the Web page http://ams.cern.ch/

[30] Navarro J F, Frenk C S and White S D M, 1996 Astrophys. J. 462563 [SPIRES]

[31] Moore B, Quinn T, Governato F, Stadel J and Lake G, 1999 Mon. Not. R. Astron. Soc. 3101147

[32] Sjöstrand T, Mrenna S and Skands P, 2006 J. High Energy Phys. JHEP05(2006)026 [SPIRES]

[33] See for example: Berezinskii V S, Buolanov S V, Dogiel V A, Ginzburg V L and Ptuskin V S, 1990 Astrophysics of Cosmic Rays (Amsterdam: North-Holland)

[34] Maurin D, Donato F, Taillet R and Salati P, 2001 Astrophys. J. 555585 [SPIRES]

[35] Delahaye T, Lineros R, Donato F, Fornengo N and Salati P, 2008 Phys. Rev. D 77063527 [SPIRES]

[36] Donato F, Maurin D and Taillet R, 2002 Astron. Astrophys. 381539 [SPIRES] [astro-ph/0108079]

[37] Hisano J, Matsumoto S, Saito O and Senami M, 2006 Phys. Rev. D 73055004 [SPIRES]

[38] Baltz E A and Edsjo J, 1999 Phys. Rev. D 59023511 [SPIRES]

[39] Moskalenko I V and Strong A W, 1998 Astrophys. J. 493694 [SPIRES] 
[40] Baltz E A, Edsjo J, Freese K and Gondolo P, 2002 Phys. Rev. D 65063511 [SPIRES]

[41] Tan L C and Ng L K, 1983 J. Phys. G: Nucl. Part. Phys. 9227 [SPIRES]

[42] Donato F, Maurin D, Salati P, Taillet R, Barrau A and Boudoul G, 2001 Astrophys. J. 563172 [SPIRES]

[43] Gleeson L J and Axford W I, 1967 Astrophys. J. 149 L115 [SPIRES]

Gleeson L J and Axford W I, 1968 Astrophys. J. 1541011 [SPIRES]

[44] Perko J S, 1987 Astron. Astrophys. 184119 [SPIRES]

[45] Moskalenko I V, Strong A W, Ormes J F and Potgieter M S, 2002 Astrophys. J. 565280 [SPIRES]

[46] in progress 